\title{
New solutions to capture and enrich bacteria from complex samples
}

\author{
Maria G. Sande ${ }^{1} \cdot$ Tugçe Çaykara $^{1,2} \cdot$ Carla Joana Silva ${ }^{2} \cdot$ Ligia R. Rodrigues $^{1} \mathbb{C}$
}

Received: 29 July 2019 / Accepted: 22 January 2020 / Published online: 5 February 2020

(c) The Author(s) 2020

\begin{abstract}
Current solutions to diagnose bacterial infections though reliable are often time-consuming, laborious and need a specific laboratory setting. There is an unmet need for bedside accurate diagnosis of infectious diseases with a short turnaround time. Moreover, low-cost diagnostics will greatly benefit regions with poor resources. Immunoassays and molecular techniques have been used to develop highly sensitive diagnosis solutions but retaining many of the abovementioned limitations. The detection of bacteria in a biological sample can be enhanced by a previous step of capture and enrichment. This will ease the following process enabling a more sensitive detection and increasing the possibility of a conclusive identification in the downstream diagnosis. This review explores the latest developments regarding the initial steps of capture and enrichment of bacteria from complex samples with the ultimate goal of designing low cost and reliable diagnostics for bacterial infections. Some solutions use specific ligands tethered to magnetic constructs for separation under magnetic fields, microfluidic platforms and engineered nano-patterned surfaces to trap bacteria. Bulk acoustics, advection and nano-filters comprise some of the most innovative solutions for bacteria enrichment.
\end{abstract}

Keywords Diagnostics $\cdot$ Bacteria capture $\cdot$ Enrichment $\cdot$ Detection limit $\cdot$ Microfluidics $\cdot$ Magnetic beads $\cdot$ Magnetic nanoparticles $\cdot$ Acoustophoresis $\cdot$ Aptamer $\cdot$ Bacteriophage $\cdot$ Peptide $\cdot$ Point-of-care $\cdot$ Nano-patterning

\section{Introduction}

Infectious diseases including lower respiratory infections, tuberculosis and diarrheal diseases are among the top ten causes of mortality globally, ahead of dementia, cancer and diabetes mellitus [1]. For instance, every year, nearly 1.7 billion cases of childhood diarrheal disease are registered, being mainly transmitted by pathogens such as Escherichia coli present in contaminated food and water [2]. The scale of diseases prevalence, multiplicity of bacterial species and strains involved and disease incidence, in addition to the emergence of opportunistic pathogens, make clinical diagnostics a challenging task, especially in places with lack of resources.

Edited by Volkhard A. J. Kempf.

Ligia R. Rodrigues

lrmr@deb.uminho.pt

1 CEB-Centre of Biological Engineering, Universidade do Minho, Campus de Gualtar, 4710-057 Braga, Portugal

2 CENTI-Center for Nanotechnology and Smart Materials, Rua Fernando Mesquita 278, 4760-034 Vila Nova de Famalicão, Portugal
Culture-based assays continue to be the gold standard for bacteria identification [3]. However, this methodology is time-consuming taking several days in some cases [4]. Moreover, highly skilled labor and a specific laboratory setting is required, which can limit their use in some places where proper facilities are not available. In the last decades, several immunological and molecular methods have been developed for pathogen diagnostics with a high level of sensitivity and accuracy. The most popular methods include immuno-based biosensors, enzyme-linked immunosorbent assay (ELISA) and molecular methods such as polymerase chain reaction (PCR), mass spectrometry (MS) and more recently, loop-mediated isothermal amplification (LAMP) and next generation sequencing (NGS) [4]. Though highly sensitive, these methods involve complex, time-consuming sample preparation steps, sophisticated laboratory equipment and highly skilled labor.

Despite of the current availability of a multitude of bacteria identification methods, there is still an enormous unmet need for fast, easy to use, cost-effective and highly sensitive point-of-care (POC) diagnostics. Capture and enrichment techniques can be achieved with compact devices, which can potentially provide bedside diagnostics. In addition, 
sometimes the number of pathogens present in biological samples are lower than the detection limits of the available methods, thus requiring additional pre-enrichment steps which add costs to the diagnostics and increase the turnaround time. Moreover, samples usually associated with low bacterial numbers (e.g., CNS fluid) or in mixed populations (e.g., stool), can benefit from a diagnostic approach using capture and enrichment of samples prior to downstream processing. This review explores the most recent developments regarding materials, methods and approaches for capturing and/or enriching bacteria from biological complex samples towards the design of more straight-forward and cost-effective clinical diagnostic methods to be used in a POC setting (Fig. 1).

\section{New materials and methods to capture and enrich bacteria from biological samples}

Many efforts have been conducted in the recent years towards the development of innovative diagnostics against several diseases. These involve the search and engineering of specific ligands (antibodies, peptides, aptamers), the design and fabrication of new materials exhibiting a panoply of relevant physicochemical features (hydrophilicity/hydrophobicity; conductivity; wettability; rigidity; roughness; flexibility; biocompatibility; among others), and the combination of different separation (affinity, molecular weight, acoustic) and detection (fluorescence, electrochemistry, absorbance) principles to enable the efficient capture of specific pathogens from complex samples (blood, urine, stool), their enrichment and qualitative and/or quantitative detection.

\section{Magnetic beads}

Aptamers can be raised against virtually any target through SELEX (Systematic evolution of ligands by exponential enrichment) [7], thus opening a number of opportunities for the development of innovative and cheap diagnostics. A POC, aptamer-mediated biosensor was developed to aid in malaria diagnosis [5]. Aptamers are oligonucleotides fragments (DNA or RNA) that bind to their targets with high affinity and specificity [6]. An aptamer that specifically recognizes the Plasmodium falciparum lactate dehydrogenase $(\mathrm{PfLDH})$ enzyme was designed and coated onto magnetic microbeads towards its magnet-guided capture and colorimetric detection on a microfluidic platform. Moreover, the biosensor developed a signal within $20 \mathrm{~min}$ of adding a development reagent and the cost of the microfluidic platforms are not expensive costing about 3 USD per cassette.

In a recent work [8], the long tail fibers (S16 LTF) of a bacteriophage that specifically binds Salmonella were immobilized onto paramagnetic beads that were further used to capture Salmonella cells from different samples.
Bacteriophages are viruses that can only live and replicate within bacteria. They infect hosts by binding to surface receptors by their long tail fibers with unmatched specificity. Due to the inherent specificity of these bacteriophage tail fiber to bind distinct bacterial species they are suitable affinity molecules. Concentrations of captured bacteria between $10^{2}$ and $10^{7} \mathrm{CFU} / \mathrm{mL}$ were detected by a change in color of the enzymatic label used. Alternatively, specific peptides against different target pathogens can be selected by Phage Display [7] and further used to modify magnetic beads leading to new diagnostics.

Most reports provide proof-of-concept studies using synthetic samples to validate their capture and enrichment models. However, the results do not always translate to a scenario where real samples are used as discussed by Bicart-See and collaborators [9]. These authors evaluated the success of bacteria capture from clinical samples. Staphylococcus aureus was isolated from articular fluid and synovial tissue using magnetic beads coated with an engineered chimeric human opsonin protein, Fc-mannose-binding lectin (FcMBL). The direct capture from clinical samples was found to be very inefficient (lower than 5\%). Masking of FcMBL binding sites by immunoglobulins and immune cells, as well as the high viscosity of complex biological samples were found to be the main reason responsible for the low capture efficiency. However, through a pre-treatment of the clinical tissue samples with hypotonic washes, hyaluronidase and proteases, FcMBL capture efficiency increased to $76 \%$.

\section{Magnetic nanoparticles}

Wang and colleagues [10] developed a technique using iron oxide magnetic nanoparticles (MNP) functionalized with aptamers to specifically bind $S$. aureus and E. coli. Additional functionalization groups involved a photosensitizer chlorin e6 for detection by fluorescence microscopy and polyethylene glycol (PEG) to improve the stability and biocompatibility of the MNPs. The ultimate goal was to provide a quick turnaround time for diagnosis of sepsis, which can be fatal if not reversed within few hours. The developed solution enabled capturing and detecting trace amounts of bacteria (10 CFU) within $1.5 \mathrm{~h}$ from mouse blood.

Similarly, MNPs containing an iron oxide magnetite core were functionalized with an amino-glycan coating [11]. These MNPs were fixed to plastic strips and used to rapidly capture bacteria directly from complex matrices by dipping the strips into the sample. The MNP fixed to the strips have hydroxyl, amino and hydrophobic regions that interact with bacterial membranes and their surface epitopes forming non-covalent electrostatic bonds similar to those found in antibody bonding. Overall, the MNP-strip capture aided with a cyclic voltammetry detection system, detected cells 

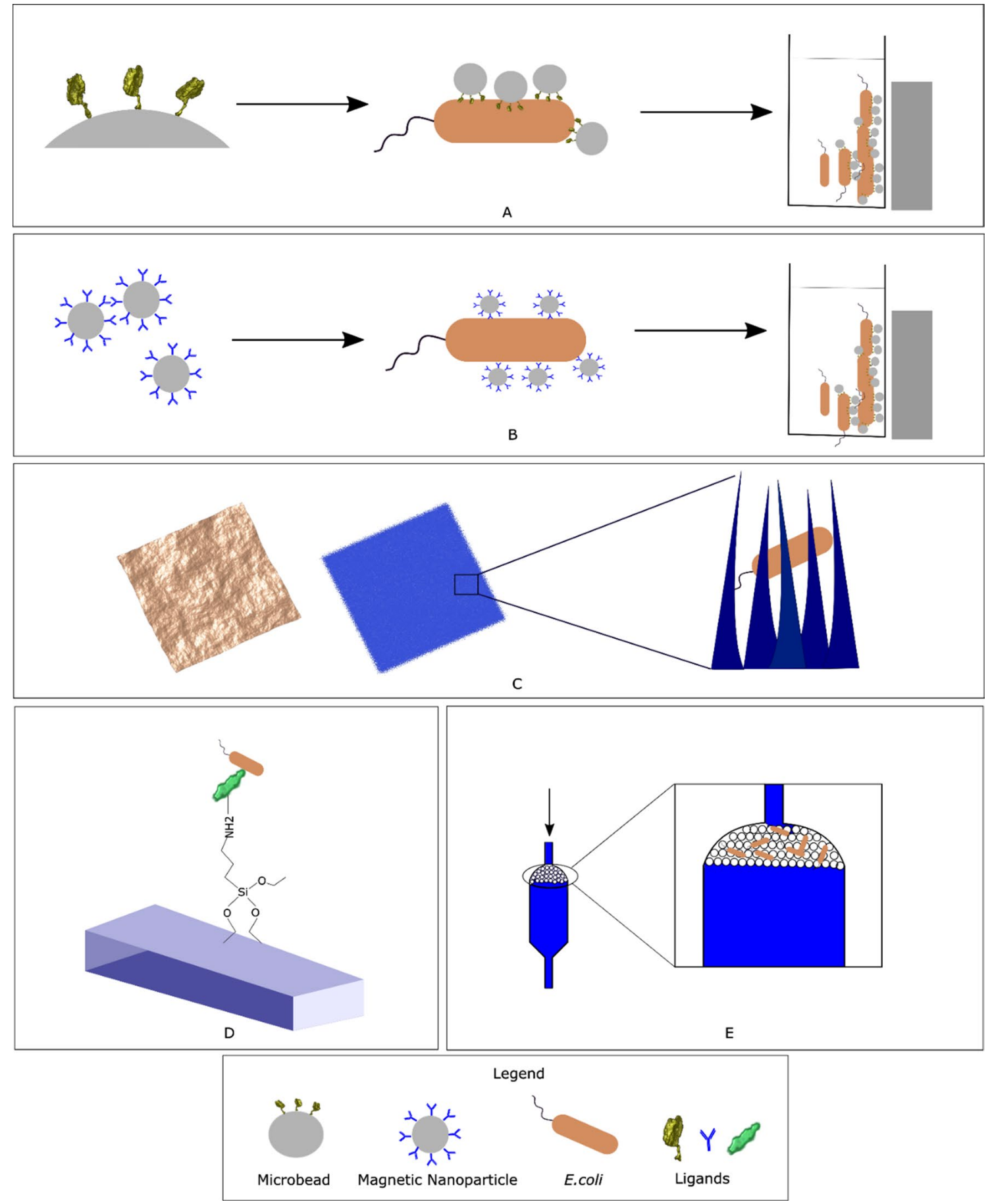

Fig. 1 Overview of the methods to capture bacteria from biological samples. a Magnetic beads functionalized with ligands that bind bacteria and can be enriched by magnetic separation. b Functionalized magnetic nanoparticles are used to bind bacteria and are enriched by magnetic separation. c Various types of nano-topographies such as prickly or nano-patterned surfaces can be engineered to trap bacte-

in less than $30 \mathrm{~min}$, without requiring an initial separation of targeted cells from the matrix. In addition, the use of plastic strips for bacteria capture significantly reduces costs. ria. d. Surfaces functionalized with chemical cross linkers and ligands that can directly capture bacteria. e Physical barriers such as nanofilters can be used to trap bacteria from a sample flowing through it. Combinations of all these methods have also been explored to capture bacteria from complex samples

In a recent development [12], a microfluidic channel was designed with a slanted ridge array that induces advective spiral flows when a sample fluid flows through the 
microfluidic channel causing enhanced separation of magnetized particles present in the sample. Cells bound with magnetic particles in the presence of the advective flow are repeatedly transferred close to a ferromagnetic nickel structure, where they can be captured easily, since the magnetic field is the strongest in the vicinity of the magnet. This system was validated using super-paramagnetic nanoparticles coated with mannose-binding lectin and bound to E. coli spiked onto undiluted whole blood from rats. $91.6 \%$ of $E$. coli bound to the MNPs were successfully separated as compared to only $23.9 \%$ of $E$. coli collected in a conventional microfluidic device.

\section{Nano-patterned 3D surfaces}

Nano-patterned 3D surfaces have higher binding capacities compared with flat substrates [13]. These surfaces offer more reactive sites to bind with bacterial targets. Several nanostructures have been evaluated regarding their ability to capture the target bacteria, including nanowires, nanofractals and nanofibers [14].

Zinc oxide nanorod arrays were fabricated by an electrochemical method to form a 3D nano-bio surface functionalized with lectin Concanavalin A (ConA) to capture E. coli [15]. Lectins are effective ligands due to their specificity of binding polysaccharide components on bacterial cells surfaces. The results showed a reasonable detection limit of $0.9 \times 10^{2} \mathrm{CFU} / \mathrm{mL}$ in saline-spiked $E$. coli.

$\mathrm{Li}$ and colleagues [14] further demonstrated the synergistic effect of 3D topography coupled with surface chemical modification to increase bacteria attachment and binding efficiency. Densely packed nanowire arrays were fabricated on silicon wafers such that the space between nanowire structures was smaller than the diameter of a bacterium to improve the binding. ConA molecules were introduced onto the nanowires through sequential chemical covalent coupling to confer selective bacteria recognition capabilities. ConA has high affinity for bacterial lipopolysaccharide components and the capture sensitivity of test assays was $10 \mathrm{CFU} / \mathrm{mL}$. The functionalized nanowires were found to capture up to ten times more $S$. aureus than the silicon-ConA substrate without nanowires and within a short incubation time of $30 \mathrm{~min}$, thus suggesting the critical role of the 3D topography in an enhanced capture of bacteria.

A dialyzer made with 3D carbon foam and grafted with nanowires was used to capture bacteria from human blood samples [16]. Pointed polycrystalline nanowires bent readily to form 3D 'nanoclaws' to trap bacteria due to a decreasing Young's moduli from the bottom to the tip resulting from sample flow. Bacteria-capture efficiency was improved from $10 \%$ on plain carbon foam and $40 \%$ on unbendable nanowires on carbon foam to $97 \%$ on bendable polycrystalline nanowires on carbon foam when placed in a simulated bloodstream flowing at $10 \mathrm{~cm} / \mathrm{s}$.

Sensors were fabricated by in situ deposition of prickly $\mathrm{Zn}-\mathrm{CuO}$ nanoparticles and graphene oxide nanosheets on an Ni porous electrode [17]. Due to burr-like nanostructures on the surface, the sensor exhibited excellent bacteria-capture efficiency (70-80\% in $20 \mathrm{~min}$ ) at low concentrations of $50 \mathrm{CFU} / \mathrm{mL}$, and high detection sensitivity with lower limit of $10 \mathrm{CFU} / \mathrm{mL}$. An excellent detection of bacteria at concentrations ranging from 10 to $10^{5} \mathrm{CFU} / \mathrm{mL}$ within a short time interval of $30 \mathrm{~min}$ was observed using an E. coli spiked rat blood sample.

\section{Methods involving specific chemicals and ligands}

Wang et al. [18] developed a multifunctional chip comprising a silicon wafer functionalized with 4-mercaptophenylboronic (4-MPBA). The 4-MPBA molecule possesses a boronic acid group that is known to reversibly bind peptidoglycan from bacterial cell walls enabling the specific capture of several bacteria. In addition, 4-MPBA has also been bound to silver nanoparticles for cell lysis and to a benzene ring that can amplify signals of the captured bacteria for their detection and identification. In terms of efficiency, $1 \times 10^{2}$ cells $/ \mathrm{mL}$ of $E$. coli or $S$. aureus were accurately identified in human blood, thus making it sensitive enough to detect bacteria in the blood of sepsis patients. Yang et al. [19] reported a similar solution using 4-MPBA on a semiconductor substrate. The platform was able to capture $E$. coli with high specificity and sensitivity with a detection limit as low as $46 \mathrm{CFU} / \mathrm{mL}$ of $E$. coli from a test suspension. Moreover, this class of SERS chip can be easily and cheaply fabricated increasing the likelihood of its use for practical applications.

A herringbone-type microfluidic platform contained a Magainin 1 peptide immobilized on its surface to enrich a test bacteria from urine samples [20]. Magainin 1 semiselectively binds to Gram-negative bacteria species. The platform was coupled with a recombinase polymerase amplification (RPA) sensor for simultaneous pathogenic DNA amplification and detection in a real time and highly sensitive manner. The detection limit was found to be 20 times more sensitive in $10 \mathrm{~mL}$ urine with Salmonella and ten times more sensitive in $10 \mathrm{~mL}$ urine with Brucella than that of real-time PCR without the enrichment step. The combination of ligand-based enrichment and an RPA sensor exhibited a detection sensitivity of $5 \mathrm{CFU} / \mathrm{mL}$ urine for $\mathrm{Sal}$ monella and $10 \mathrm{CFU} / \mathrm{ml}$ urine for Brucella within $60 \mathrm{~min}$.

\section{Methods involving sound wave acoustophoresis}

A number of microfluidic separation methods have been used to separate bacteria from the bulk sample such as 
centrifugal microfluidics and dielectrophoresis [21]. Acoustophoresis has recently been demonstrated as an effective technique to separate components from a bulk fluid in the presence of sound waves into separate streams [21]. This method has proven to be useful in sepsis diagnosis due to the quick turnaround time and possibly for blood disinfection given that it can be scaled-up to handle larger volumes.

In a recent example [22], bulk acoustophoresis was used to separate bacteria from the blood cells from whole blood samples. The small size of bacteria prevents them from being affected by an acoustic field, and therefore, they remain in the blood plasma flow that is directed to a separate outlet. Upon optimization of the sample volume, it was found that $1 \mathrm{ml}$ of undiluted whole blood can be processed within $12.5 \mathrm{~min}$, with a bacteria recovery of $90 \%$ and almost $100 \%$ blood cell removal.

In addition, bulk acoustophoresis was used to identify bacteria using a bacteriophage-based luminescence assay in a microfluidic chip [23]. To demonstrate the effectiveness of the acoustophoretic system, three clinically relevant species of bacteria were tested, namely, Pseudomonas aeruginosa, $S$. aureus and E. coli. Bacteria recovery from blood samples was between $45 \%$ and $60 \%$ across species while removing more than $85 \%$ of blood cells.

\section{Methods involving physical barriers}

Novel microfluidic immune-affinity columns consisting of packed microbeads functionalized with antibodies were used to provide large surface areas for effective capture of uropathogenic E. coli [24]. Due to the capillary action of the channels in the columns, the sample, antibody and fluorescent label were dispensed autonomously and the assay time was very fast (7 min). The detection limit of E. coli in synthetic urine was $1.2 \times 10^{2} \mathrm{CFU} / \mathrm{mL}$, which is well below the cutoff of $10^{5} \mathrm{CFU} / \mathrm{mL}$ of a single organism which is often used as a cutoff point in the diagnosis of uncomplicated urinary tract infections.

Another device was fabricated based on the self-assembly of polystyrene microparticles at a pillar array region within a microfluidic chip to form a nanoscale filter for bacteria capture from samples flowing on gold nano/micro islands [25]. The nanosurface microfluidic device showed an average capture efficiency of $93 \%$ E. coli from samples.

\section{Methods involving mixed separation principles}

PEG hydrogel bead-like scaffolds were functionalized with aptamers to specifically target $S$. aureus and E. coli. PEG is used to decrease non-specific adsorption of other targets. This PEG scaffold was tagged with MNPs to simplify the processing of the bioassays. In addition, the scaffolds were nano-patterned to increase the surface area for probe immobilization and greatly enhance binding efficiency of target bacteria. Multiple bacterial species were captured from human blood spiked with $S$. aureus and $E$. coli with concentrations as low as $100 \mathrm{CFU} / \mathrm{mL}$ within $2.5 \mathrm{~h}$ [26].

A reusable supramolecular platform was developed for the specific capture and release of bacteria and proteins [27]. The platform exploited the carbohydrate-protein affinity recognition to capture specific proteins and bacteria. It used multi-layering polyelectrolyte films, followed by the incorporation of $\beta$-cyclodextrin ( $\beta$-CD) derivatives modified with mannose as host molecules which were demonstrated to bind type I fimbriae $E$. coli and lectin proteins. The special feature of this platform is that captured bacteria could be released from the surface by incubation with sodium dodecyl sulfate and the platform can then be reused. This versatile and reusable system is very promising and could comprise the basis for the development of new, cheap and reusable diagnostic devices.

\section{Conclusion and outlook}

Classical methods used for bacterial infection diagnosis are reliable, however, in an era where faster, more convenient and flexible solutions are constantly demanded, the realm of diagnostics is no exception. There is a clear need for faster results using simple and cheap miniaturized platforms. Innovators in the diagnostics field have been inspired by the semiconductor industry to develop microfluidic devices and 'chips' to capture, detect and quantify diverse microorganisms. The great advantage of these microfluidic devices and compact sensors is that they are cheaply manufactured, costing as little as a few USD in many cases reducing the overall costs. Other cost-saving examples include the use of materials such as polystyrene and paper to manufacture some of the innovative devices herein described, or the use of aptamers as specific ligands, which are cheap and easy to synthesize.

In addition, relevant contributions from the nanotechnology and synthetic biology fields led to the development of targeted micro/nanoparticles for capture and detection purposes. Immunoassays, chemical ligand-mediated capture and functionalized magnetic microbeads or nanoparticles are among the successful solutions developed to capture bacterial cells in microfluidic devices. As a result, turnaround times have been reduced from many hours to minutes in some cases and miniaturization promises fast, sensitive and easy to use POC diagnostic devices. However, before these new solutions translate into an important improvement in the infection diagnostics field, they must be tested beyond the developmental stage. One relevant challenge includes validating these new solutions using patient samples (blood, urine, stool) that are more complex and heterogeneous than 
the synthetic samples commonly used at the developmental stage. Therefore, it is always important to bear in mind that even if a diagnostic method performs very well with synthetic samples, it might need further adjustments and optimizations before it can be used in a real context with biological samples. In addition, while in theory, these new solutions appear to be cost-effective, some use expensive ligands (e.g., antibodies) and, for instance, the design of complex and sophisticated microfluidic platforms can also significantly increase costs. Other solutions being proposed bypass the analyte-ligand binding making use of nano-patterned surfaces or micro/nano-obstacles and filters to capture bacteria. However, these solutions may lack specificity. An alternative approach could be to use inexpensive ligand biomolecules (e.g., aptamers) immobilized onto available and common polymeric substrates (e.g., silicon), thereby taking advantage of the specificity and sensitivity of the ligand coupled to a cheap platform. The development of these innovative solutions will undoubtedly lead to a new generation of tools to capture and detect bacteria, thus making POC diagnosis of an infection within hours or even minutes and overcoming the limitations of the conventional methods, a reality.

Funding This research is affiliated to the VibrANT project that received funding from the EU Horizon 2020 Research and Innovation Programme under the Marie Sklowdowska-Curie Grant, agreement no 765042. In addition, MGS, TC and LRR acknowledge the financial support from Fundação para a Ciência e Tecnologia (FCT) under the scope of the strategic funding of UID/BIO/04469/2020 unit and COMPETE 2020 (POCI-01-0145-FEDER-006684), through national funds and where applicable co-financed by the FEDER, within the PT2020 Partnership Agreement. The same authors also acknowledge BioTecNorte operation (NORTE-01-0145-FEDER-000004) funded by the European Regional Development Fund under the scope of Norte2020-Programa Operacional Regional do Norte.

\section{Compliance with ethical standards}

Conflict of interest The authors declare no conflict of interest.

Open Access This article is licensed under a Creative Commons Attribution 4.0 International License, which permits use, sharing, adaptation, distribution and reproduction in any medium or format, as long as you give appropriate credit to the original author(s) and the source, provide a link to the Creative Commons licence, and indicate if changes were made. The images or other third party material in this article are included in the article's Creative Commons licence, unless indicated otherwise in a credit line to the material. If material is not included in the article's Creative Commons licence and your intended use is not permitted by statutory regulation or exceeds the permitted use, you will need to obtain permission directly from the copyright holder. To view a copy of this licence, visit http://creativecommons.org/licenses/by/4.0/.

\section{References}

1. WHO (2015) The top 10 causes of death. http://who.int/media centre/factsheets/fs310/en/. Accessed 1 July 2019

2. Word Health Organization (2013) Diarrhoeal disease 2013. https ://www.who.int/news-room/fact-sheets/detail/diarrhoeal-disease. Accessed 1 July 2019

3. Fournier PE, Dubourg G, Raoult D (2014) Clinical detection and characterization of bacterial pathogens in the genomics era. Genome Med 6:1-15

4. Zhang D, Bi H, Liu B, Qiao L (2018) Detection of pathogenic microorganisms by microfluidics based analytical methods. Anal Chem 90:5512-5520

5. Fraser LA, Kinghorn AB, Dirkzwager RM, Liang S, Cheung YW, Lim B, Shiu SCC, Tang MSL, Andrew D, Manitta J, Richards JS, Tanner JA (2018) A portable microfluidic Aptamer-Tethered Enzyme Capture (APTEC) biosensor for malaria diagnosis. Biosens Bioelectron 100:591-596

6. Templier V, Roux A, Roupioz Y, Livache T (2016) Ligands for label-free detection of whole bacteria on biosensors: a review. TRAC Trends Anal Chem 79:71-79

7. Rodrigues JL, Ferreira D, Rodrigues LR (2017) Synthetic biology strategies towards the development of new bioinspired technologies for medical applications. In: Rodrigues LR, Mota M (eds) Bioinspired materials for medical applications. WoodHead Publishing, Elsevier, Amsterdam

8. Dunne M, Loessner MJ (2019) Modified bacteriophage tail fiber proteins for labeling, immobilization, capture, and detection of bacteria. Methods Mol Biol 1918:67-86

9. Bicart-See A, Rottman M, Cartwright M, Seiler B, Gamini N, Rodas M, Penary M, Giordano G, Oswald E, Super M, Ingber DE (2016) Rapid isolation of Staphylococcus aureus pathogens from infected clinical samples using magnetic beads coated with Fc-mannose binding lectin. PLoS One 11:1-12

10. Wang J, Wu H, Yang Y, Yan R, Zhao Y, Wang Y, Chen A, Shao S, Jiang P, Li YQ (2018) Bacterial species-identifiable magnetic nanosystems for early sepsis diagnosis and extracorporeal photodynamic blood disinfection. Nanoscale 10:132-141

11. Matta LL, Harrison J, Deol GS, Alocilja EC (2018) Carbohydratefunctionalized nanobiosensor for rapid extraction of pathogenic bacteria directly from complex liquids with quick detection using cyclic voltammetry. IEEE Trans Nanotechnol 17:1006-1013

12. Jung SH, Hahn YK, Oh S, Kwon S, Um E, Choi S, Kang JH (2018) Advection flows-enhanced magnetic separation for highthroughput bacteria separation from undiluted whole blood. Small $14: 1-7$

13. Wang S, Zhang P, Liu H, Jiang L, Xu T, Meng J, Liu X, Yang G, Chen L (2013) Programmable fractal nanostructured interfaces for specific recognition and electrochemical release of cancer cells. Adv Mater 25:3566-3570

14. Li YQ, Zhu B, Li Y, Leow WR, Goh R, Ma B, Fong E, Tang M, Chen X (2014) A synergistic capture strategy for enhanced detection and elimination of bacteria. Angew Chemie Int 53:5837-5841

15. Zheng L, Wan Y, Qi P, Sun Y, Zhang D, Yu L (2017) Lectin functionalized $\mathrm{ZnO}$ nanoarrays as a 3D nano-biointerface for bacterial detection. Talanta 167:600-660

16. Liu L, Chen S, Xue Z, Zhang Z, Qiao X, Nie Z, Han D, Wang J, Wang T (2018) Bacterial capture efficiency in fluid bloodstream improved by bendable nanowires. Nat Commun 9:1-9

17. Wu R, Ma Y, Pan J, Lee SH, Liu J, Zhu H, Gu R, Shea KJ, Pan G (2018) Efficient capture, rapid killing and ultrasensitive detection of bacteria by a nano-decorated multi-functional electrode sensor. Biosens Bioelectron 101:52-59

18. Wang H, Zhou Y, Jiang X, Sun B, Zhu Y, Wang H, Su Y, He Y (2015) Simultaneous capture, detection, and inactivation of 
bacteria as enabled by a surface-enhanced Raman scattering multifunctional chip. Angew Chemie Int 54:5132-5136

19. Yang Z, Wang Y, Zhang D (2018) An integrated multifunctional photoelectrochemical platform for simultaneous capture, detection, and inactivation of pathogenic bacteria. Sens Actuator B Chem 274:228-234

20. Dao TNT, Lee EY, Koo B, Jin CE, Lee TY, Shin Y (2018) A microfluidic enrichment platform with a recombinase polymerase amplification sensor for pathogen diagnosis. Anal Biochem 544:87-92

21. Pitt WG, Alizadeh M, Husseini GA, McClellan DS, Buchanan CM, Bledsoe CG, Robison RA, Blanco R, Roeder BL, Melville M, Hunter AK (2016) Rapid separation of bacteria from bloodreview and outlook. Biotechnol Prog 32:823-839

22. Ohlsson P, Petersson K, Augustsson P, Laurell T (2018) Acoustic impedance matched buffers enable separation of bacteria from blood cells at high cell concentrations. Sci Rep 8:1-11

23. Dow P, Kotz K, Gruszka S, Holder J, Fiering J (2018) Acoustic separation in plastic microfluidics for rapid detection of bacteria in blood using engineered bacteriophage. Lab Chip 18:923-932
24. Olanrewaju AO, Ng A, Decorwin-Martin P, Robillard A, Juncker D (2017) Microfluidic capillaric circuit for rapid and facile bacteria detection. Anal Chem 89:6846-6853

25. Abdelfatah T, Jalali M, Mahshid S (2018) A nanosurface microfluidic device for capture and detection of bacteria. IEEE Life Sci Conf LSC 2018:167-170

26. Xu Y, Wang H, Luan C, Liu Y, Chen B, Zhao Y (2018) Aptamerbased hydrogel barcodes for the capture and detection of multiple types of pathogenic bacteria. Biosens Bioelectron 100:404-410

27. Qu Y, Wei T, Zhan W, Hu C, Cao L, Yu Q, Chen H (2017) A reusable supramolecular platform for the specific capture and release of proteins and bacteria. J Mater Chem B 5:444-453

Publisher's Note Springer Nature remains neutral with regard to jurisdictional claims in published maps and institutional affiliations. 